\title{
LXII. On the general theory of duplex telegraphy
}

\section{Louis Schwendler}

To cite this article: Louis Schwendler (1876) LXII. On the general theory of duplex telegraphy , Philosophical Magazine Series 5, 1:7, 526-542, DOI: 10.1080/14786447608639077

To link to this article: http://dx.doi.org/10.1080/14786447608639077

曲 Published online: 13 May 2009.

Submit your article to this journal 준

Џll Article views: 2

Q View related articles $₫$ 


\section{On the General Theory of Duplex Telegraphy.} $B y$ Louis Schw ENDLER*

[Continued from S. 4. vol. 1. p. 475.]

\section{The Compensation Method $\uparrow$.}

THIS method is the oldest; fig. 3 gives the general diaI. gram.

Fig. 3.

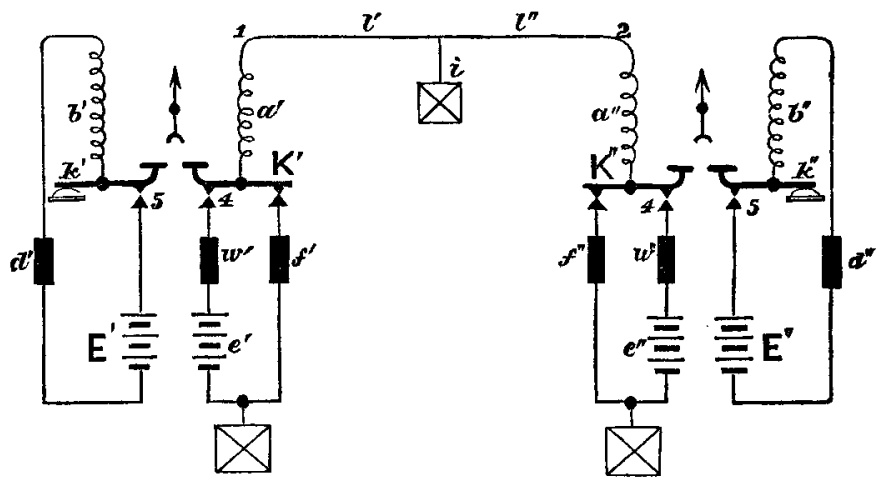

$e$ is the electromotive force of the line battery.

$\beta$ its internal resistance.

$\mathbf{E}$ is the electromotive force of the compensation battery.

a its internal resistance.

$\mathrm{K}$ is a constant-resistance key. Dr. Gintl used an ordinary key, whieh, it will be obvious, must result in a failure.

$k$ is an ordinary key : both keys, in the same station, are worked simultaneously; $i$. e. contacts 4 and 5 are closed and broken at one and the same time.

$d, f$, and $w$ are certain resistances.

$a$ is the one coil of the differential instrument, which is connected up in the line-circuit.

$b$ is the other coil of the differential instrument, which is connected up in the compensation circuit. By $a$ and $b$ shall be also designated the resistances of these two coils.

The coils $a$ and $b$ with their batteries $e$ and E respectively are arranged in such a manner that they have opposite magnetic effects with respect to the same magnetic pole. The two circuits in each station (the line-circuit and the compensation circuit) are insulated from each other. All the other terms, as $\mathrm{L}, \mathrm{L}^{\prime}, \mathrm{L}^{\prime \prime}$, \&c., shall have the same physical meaning as before.

* Communicated by the Author from the Journal of the Asiatic Society of Bengal, vol. xliv. part 2, 1875 .

$\dagger$ Dr. Wilhelm Gintl, $k .-k$. Director-General of Telegraphs in Austria, is the inventor of this earliest method. In 1853 he made the first practical experiment on a line between Vienna and Prague (240 miles). 
The compensation method has two principal defects which the two preceding methods do not possess.

First. The success of working a line dupliciter by the compensation method will clearly depend on the possibility of being able to close and open simultaneously two different contacts $(4$ and 5$)$. The mechanical difficulty of doing so sufficiently accurately was pointed out by Dr. Werner Siemens, and in fact constitutes one of the reasons which led him to propose the differential method.

Secondly. The balance in each station may be disturbed directly by a variation of the electrical condition (internal resistance and electromotive force) of the two batteries (E and e) employed.

In the preceding two methods the variation of the internal resistance of the signalling-battery can only be felt indirectly by affecting the balance of the distant station, while the variation of electromotive force has no effect at all. Hence a given variation in the battery or batteries must necessarily produce a greater disturbance of balance in the compensation method than in the two preceding ones. We know that even so-called constant galvanic batteries, doing work, alter their electrical conditions perceptibly, especially their internal resistance; and consequently this defect weighs most decidedly against the compensation method. In all other respects the compensation method has the same defects as the differential method, and in addition some others which will be understood as the investigation proceeds.

\section{General expression for the two functions "D" and "S."}

To obtain the functions $\mathrm{D}$ and $\mathrm{S}$, we have to develop the general expressions for the forces $p, \dot{\mathrm{P}}$, and $\mathrm{Q}$, say (for station I.)

$$
p^{\prime}=\mathrm{A}^{\prime} m^{\prime}-\mathrm{B}^{\prime} n^{\prime}
$$

where $\mathrm{A}^{\prime}$ and $\mathrm{B}^{\prime}$ are the currents which pass through the two coils $a^{\prime}$ and $b^{\prime}$ respectively when station $I$. is sending and station II. is at rest; $m^{\prime}$ and $n^{\prime}$ are the forces exerted by the two coils $a^{\prime}$ and $b^{\prime}$ respectively on one and the same magnetic pole when a unit of current passes through them. At balance in station I.

Further,

$$
p^{\prime}=0 \text {. }
$$

$$
\mathrm{P}^{\prime}=\mathfrak{A}^{\prime} m^{\prime},
$$

where $\mathfrak{A}^{\prime}$ is the current which passes through the coil $a^{\prime}$ when station II. is sending and station I. is at rest (single signals). Further,

$$
\mathrm{Q}^{\prime}=\mathrm{V}^{\prime} m^{\prime}+\mathrm{G}^{\prime} n^{\prime}
$$


where $\mathrm{F}^{\prime}$ and $\mathrm{G}^{\prime}$ are the currents which pass through the coils $a^{\prime}$ and $b^{\prime}$ respectively when both stations are sending simultaneously (duplex signals).

The compensation circuit and the line-circuit in each station being electrically independent of each other, we have

invariably without condition.

$$
\mathrm{g}^{\prime}=\mathrm{B}^{\prime}
$$

If we further presuppose that depressing of the key $K$ does not alter the complex resistance of the station (a condition which, for the regularity of signals, we are obliged to assume here as well as in the two preceding methods), it will be clear that

$$
\nabla^{\prime}=A^{\prime}+{ }^{\prime} \text {. }
$$

Substituting these values for $\mathrm{V}^{\prime}$ and $\mathrm{g}^{\prime}$ in the expression for $\mathrm{Q}^{\prime}$, we get

$$
\begin{aligned}
& p^{\prime}=\mathrm{A}^{\prime} m^{\prime}-\mathrm{B}^{\prime} n^{\prime}, \\
& \mathrm{P}^{\prime}=\mathfrak{A}^{\prime} m^{\prime}, \\
& \mathrm{Q}^{\prime}=\left(\mathrm{A}^{\prime}+\mathfrak{A}^{\prime}\right) m^{\prime}+\mathrm{B}^{\prime} n^{\prime} .
\end{aligned}
$$

The signs of the terms may be again contained in the currents, while $m^{\prime}$ and $n^{\prime}$ are taken as absolute numbers. We must only remember that $\mathrm{A}^{\prime} m^{\prime}$ and $\mathrm{B}^{\prime} n^{\prime}$ must be invariably of opposite sign. Arbitrarily we will call the current A positive when the negative pole of the line-battery is to earth.

Now we have again two different modes of connecting up the line-batteries, viz. :-

1st. The same poles of the line-batteries are connected to earth in the two stations:

$$
\begin{aligned}
& p^{\prime}= \pm \mathrm{A}^{\prime} m^{\prime} \mp \mathrm{B}^{\prime} n^{\prime}, \\
& \mathrm{P}^{\prime}=\mp \mathfrak{A}^{\prime} m^{\prime}, \\
& \mathrm{Q}^{\prime}=\left( \pm \mathrm{A}^{\prime} \mp \mathfrak{A}^{\prime}\right) m^{\prime} \mp \mathrm{B}^{\prime} n^{\prime} .
\end{aligned}
$$

2nd. Opposite poles of the two line batteries are connected to earth in the two stations:

$$
\begin{aligned}
& p^{\prime}= \pm A^{\prime} m^{\prime} \mp \mathrm{B}^{\prime} n^{\prime}, \\
& \mathrm{P}^{\prime}= \pm \mathfrak{A}^{\prime} m^{\prime}, \\
& \mathrm{Q}^{\prime}=\left( \pm \mathrm{A}^{\prime} \pm \mathfrak{A}^{\prime}\right) m^{\prime} \mp \mathrm{B}^{\prime} n^{\prime} .
\end{aligned}
$$

Subtracting in either case $\mathrm{P}^{\prime}$ from $\mathrm{Q}^{\prime}$, we get

$$
\mathrm{Q}^{\prime}-\mathrm{P}^{\prime}=\mathrm{S}^{\prime}=p^{\prime}
$$

Or, on account of having fulfilled the key-equation $f=w+\beta$, the difference of the forces which produce single and duplex signals is equal in sign and magnitude to the force by which balance is disturbed. Further, it is also, for the compensation 
method, quite immaterial whether the same or opposite poles of the two line-batteries are connected to earth. As pointed out, it is preferable to connect the same poles, $i$. e. the negative poles, of the line-batteries to earth.

Assuming this case, we have

$$
\begin{aligned}
& p^{\prime}=\mathrm{A}^{\prime} m^{\prime}-\mathrm{B}^{\prime} n^{\prime}, \\
& \mathrm{P}^{\prime}=-\mathbf{A}^{\prime} m^{\prime}, \\
& \mathrm{Q}^{\prime}=\left(\mathrm{A}^{\prime}-\boldsymbol{A}^{\prime}\right) m^{\prime}-\mathrm{B}^{\prime} n^{\prime} .
\end{aligned}
$$

Substituting now for $A^{\prime}, B^{\prime}$, and $\mathfrak{A}^{\prime}$ their values, and remembering that

$$
\left.\begin{array}{rl}
m^{\prime} & =q^{\prime} \sqrt{a^{\prime}}, \\
n^{\prime} & =r^{\prime} \sqrt{b^{\prime}}
\end{array}\right\} \text { approximately, }
$$

we get the following general expressions for the two functions $\mathrm{D}$ and $\mathrm{S}:-$

and

$$
\left.\begin{array}{l}
\mathrm{S}^{\prime}=e^{\prime} q^{\prime} \frac{\Delta^{\prime}}{\overline{\mathrm{R}^{\prime} \mathrm{K}^{\prime}}}, \\
\mathrm{D}^{\prime}=\frac{e^{\prime}}{e^{\prime \prime}} \cdot \frac{\mathrm{K}^{\prime \prime}}{\overline{\mathrm{R}}^{\prime} \mathrm{K}^{\prime}} \cdot \frac{\Delta^{\prime}}{\mu^{\prime} \sqrt{a^{\prime}}}
\end{array}\right\} \text { for station I. }
$$

where

$$
\left.\begin{array}{l}
\mathrm{S}^{\prime \prime}=e^{\prime \prime} q^{\prime \prime} \frac{\Delta^{\prime \prime}}{\mathrm{R}^{\prime \prime} \mathrm{K}^{\prime \prime}}, \\
\mathrm{D}^{\prime \prime}=\frac{e^{\prime \prime}}{e^{\prime}} \cdot \frac{\mathrm{K}^{\prime}}{\mathrm{R}^{\prime \prime} \mathrm{K}^{\prime \prime}} \cdot \frac{\Delta^{\prime \prime}}{\mu^{\prime \prime} \sqrt{a^{\prime \prime}}},
\end{array}\right\} \text { for station II., }
$$

$$
\begin{aligned}
\Delta & =\mathrm{R} \sqrt{a}-\mathrm{K} \lambda v \sqrt{b}, \\
\mathrm{R} & =\alpha+b+d, \\
\mathrm{~K} & =f+a+c, \\
\lambda & =\frac{\mathbf{E}}{\boldsymbol{e}} \\
v & =\frac{\boldsymbol{r}}{q}
\end{aligned}
$$

Rigid fulfiment of the two functions $\mathrm{S}=0$ and $\mathrm{D}=0$.

For finite quantities these two functions can only become zero if $\Delta=0, i . e$.

$$
\mathrm{R} \sqrt{a}-\mathrm{K} \lambda v \sqrt{b}=0,
$$

which is the balance-equation for the compensation method.

To fulfil this equation permanently, no matter what the special cause of disturbance may be, we can again adopt two ess entially different modes of readjustment, viz. :-

Either leave the two coils $a$ and $b$ or their armatures staPhil. Mag. S. 5. No. 7. Suppl. Vol. 1. 
tionary, and adjust balance by altering the resistance in one or both of the two circuits, or leave the resistances constant and alter the relative position of the two coils or their armatures with respect to a given magnetic pole. These two methods of readjusting balance shall be considered separately.

a. Readjustment of balance by altering resistances.

In order to have immediate balance it will be clear that the alteration of resistance must be restricted to the compensation circuit, which is electrically independent of the line-circuit. The total resistance in the compensation circuit consists of three different resistances, namely $b, a$, and $d$. Neither $b$ nor $\alpha$, considering their nature, can conveniently be made adjustable in practice; hence the alteration of resistance in the compensation circuit is restricted to $d$, which must therefore consist of increments of the proper size. The adjustment of $d$ should be quick and convenient.

In addition to this adjustment, $\lambda=\frac{\mathrm{E}}{e}$ may be made adjustable by varying $\mathrm{E}$ in increments of one cell. Such an adjustment, however, is not fine enough for ordinary use ; the electromotive force of one cell is too large a quantity in comparison with the total electromotive force used in the compensation circuit. If the variation of the line-current becomes very great, it might perhaps be found convenient to alter $\mathrm{E}$; but as an ordinary mode of adjustment it must be dispensed with *.

It is scarcely needed to point out that to adjust balance by altering the line-current, either by varying the resistance or the electromotive force $\dagger^{\dagger}$ or both of the line-circuit, must be rejected once for all, because such an adjustment of balance in the one station could never take place without disturbing the balance of the other station, or, in other words, the required immediate balance could not be fulfilled.

b. Readjustment of balance by moving the coils or armatures.

If we suppose both the coils or their armatures simultaneously movable in the same direction, then clearly this mode of adjustment contains not only the required immediate balance, but in addition represents also a very rapid and entirely continuous action. For this reason it is apparently preferable to

* During the period of low insulation of the line it might be advisable and practicable to make $E$ larger than during the period of high insulation of the line (wet and dry sersons).

$\dagger$ Alteration of electromotive force of a galvanic battery cannot be achieved without altering its internal resistance. Hence varying $e$ would also involve a variation of $\beta$; and in order to $\operatorname{keep} f=w+\beta$, it would become necessary to alter $w$ simultaneously with $e$; i. e. $w$ would hare to be increased when $e$ decreases, and rice versa. This method, besides being rough, would therefore be also inconvenient. 
the first method, where the adjustment can only be carried on in one branch by varying $d$ in increments*. Which of the two methods, however, is to be finally chosen, depends on other considerations, which will become clear further on. We know now that both these modes of adjustment are convenient and practicable, and contain immediate balance without special conditions. In fact in this respect the compensation method is preferable to the differential method, where immediate balance by varying resistances could only be obtained when varying the four branches simultaneously according to a fixed relation.

\section{Rapid approximation of the two functions $\mathrm{S}$ and $\mathrm{D}$ towards zero.}

On account of $f=w+\beta$ we have

where

$$
\mathrm{S}=p=e q \frac{\Delta}{\mathrm{RK}}
$$

$$
\Delta=\mathbf{R} \sqrt{a}-\mathrm{K} \lambda v \sqrt{b}
$$

Now suppose $\Delta=0$; then this equation may be disturbed by $\mathbf{K}, \mathbf{R}, \lambda, v, a$, or $b$ varying. $a$ and $b$ are wire resistances which

* It has been suggested to adjust balance by a continuous variation of resistance, as, for instance, by moving a contact-point along a thin platinum wire in the same manner as Dr. W. Siemens has done it in his bridge employed for comparing accurately comparatively small resistances. It is, however, scarcely necessary to point out that such a method, if applied for duplex working, must result in a failure, at all events so long as electromagnetic instruments are used for producing the signals. For in such a case the resistance of any branch, no matter what special duplex method may be employed, must bear a certain ratio to the given resistance of the line in order to get the signals with sufficient force. This ratio, as my investigations have shown, is by no means a small one; and hence the resistances of all branches, even for a short line, cannot be made small. Therefore the platinum wire constituting part of one or two branches of the duplex method employed, must also offer a considerable resistance, $i$. e. must be of great length. Hence to alter such a large resistance continuously and perceptibly, as is indicated by the balance-disturbance, must evidently involve a considerable movement of the contact-point, which, even choosing the thinnest possible wire and the shortest telegraph-line, becomes already for the daily variation so large as to make its application impossible. Unless another material of much higher specific resistance than platinum wire can be found, which at the same time allows of the sliding contact being made securely, the adjustment of balance by a continuous variation of resistances must be dispensed with. Such a material does not appear to exist. I thought of acting on Phillips's suggestion to use pencil-marks for the adjustable resistance; and although I found that pencil resistances can be adjusted very accurately, and can be enclosed in a very small space, and that they keep sufficiently constant, it is difficult, if not impossible, to alter them by a sliding contact; the "Uebergangswiderstand" is too variable and too great. Besides, if the contact is made with sufficient pressure, its sliding along alters the thickness of the pencilmark, and hence the resistances become inconstant and uncertain. 
may be taken as constant, for their variation with temperature is exceedingly small; and in case of accident (i. e. a coil breaking or becoming shunted) nothing short of actual repair could help. Further, $v$, supposing the differential instrument to be properly designed and mechanically well executed, may be taken as a perfectly constant quantity, which certainly, as long as the coils or their armatures are not moved on purpose, does not alter of its own accord.

The quantities left, which by variation may affect the balanceequation, are $\mathrm{K}, \mathrm{R}$, and $\lambda$.

Of these three quantities the variation of $K$ may become largest; for $\mathrm{K}$ does not only contain the line-resistance, which is highly variable, but $\mathrm{K}$ includes also the internal resistance of both the line-batteries, which, even for the best known form of galvanic battery, is by no means a constant quantity. The variation of the internal resistance of the line-battery in each station produces, of course, the greatest disturbance of balance in that station.

The next quantity most liable to change of its own accord is clearly $R$, since it contains the internal resistance of the compensation battery.

$\lambda$, the ratio of the two electromotive forces in one and the same station, though also liable to change, will yet vary very little. The electromotive force of a well-prepared galvanic battery, especially when the battery is worked by weak currents, is far more constant than is generally believed*.

With respect to the variation of the three quantities $K, R$, and $\lambda$, the function $S$ may therefore be expressed in three different forms.

$$
\begin{aligned}
& \mathrm{S}_{1}=e q \frac{\lambda v \sqrt{b}}{\mathrm{RK}} \delta \mathrm{K} \text { when } \mathrm{K} \text { alone varies. } \\
& \mathrm{S}_{2}=e q \frac{\sqrt{a}}{\mathrm{RK}} \delta \mathrm{R} \text { when } \mathrm{R}, \mathrm{i} \text {. e. } \alpha \text {, alone varies. } \\
& \mathrm{S}_{3}=e q \frac{v \sqrt{b}}{\mathrm{R}} \delta \lambda \text { when } \lambda, \text { i. e. } \mathrm{E} \text { or } e \text { or both, alone vary. }
\end{aligned}
$$

These three different disturbances of balance may act singly or conjointly; and it is clear that they are independent of each

* It appears that changes which have been observed to take place in the electromotive force of a Minotto or Leclanche's battery are generally apparent only, not real. Such changes are generally quite within the limits of observation-errors; and if they are large, they are then generally due to the incorrectness of the method employed for measuring the electromotive force, or to cells actually having become exhausted. It appears that this mysterious force in each cell either exists in its full vigour or not at all; there seems to be no continuous change in either direction. 
other, at all events as far as this investigation is concerned. Consequently the safest plan will be to make each influence as small as the circumstances will allow it.

The disturbance $\mathrm{S}_{1}$ for any constant $e q \lambda v \sqrt{b}$ and any given $\delta \mathrm{K}$ will obviously become smaller the larger $\mathrm{RK}$ is selected. Supposing $R+K$ constant, whatever that value finally may be, $\mathrm{RK}$ has a maximum for $\mathrm{R}=\mathrm{K}$; and the very same condition will obviously make the disturbance $\mathrm{S}_{2}$ smallest.

$\mathrm{S}_{3}$ offers no best condition; this expression only shows that it has an absolute maximum with respect to $b$, namely (as $\mathrm{R}=\alpha+d+b)$ for $b=\alpha+d$.

Thus we are informed that whatever relation between $b$ and $\alpha+d$ may be finally chosen, $b=\alpha+d$ should not be selected, as otherwise any given variation of $\lambda$ would have the greatest possible disturbing effect on the balance. But $b=\alpha+d$ being the condition for the maximum magnetic effect in the compensation circuit, it is hereby established that for the sake of regularity of signals, which under all circumstances is to be considered of paramount importance in duplex telegraphy, the magnetic effect in the compensation branch must not be achieved in the most economical manner, but quite the reverse. This, as the compensation circuit has actually to produce wholly or partly the duplex signals, is a testimonium paupertatis for the compensation method, and proves it in this respect inferior to both the double balance and the differential method.$$
\mathrm{R}=\mathrm{K}
$$

is the regularity condition for the compensation method; $\imath$. $e$.

In order to make the disturbance of balance by a variation of the resistance in both the circuits absolutely as small as possible, the total resistance of the compensation circuit should be equal to the total resistance of the line-circuit* .

If we now substitute in $S_{1}$ for $K$ the value $R$, and in $S_{2}$ for $R$ the value $K$, we get

$$
\begin{aligned}
\mathrm{S}_{1} & =e q \frac{\lambda v \sqrt{b}}{\mathrm{R}^{2}} \delta \mathrm{K}, \\
\mathrm{S}_{2} & =e q \frac{\sqrt{a}}{\mathrm{~K}^{2}} \delta \mathrm{R}
\end{aligned}
$$

* This result is against the adopted view, for Dr. Gintl, as well as others after him, has always treated the compensation circuit as a kind of local circuit, i. e. giving to it as low a resistance as practice allows. But this is clearly wrong; for if $R$ is made very small as compared with $K$, the balance becomes unstable. This fact explains, to a certain degree, the failure which has attended the application of the compensation method for duplex working, because the method was tried under the most unfavourable quantitative arrangements. 
while

$$
\mathbf{S}_{\mathrm{g}}=e \frac{v \sqrt{b}}{\mathbf{R}} \delta \lambda
$$

remains the same.

$\mathrm{S}_{1}$ has an absolute maximum for $b=\frac{a+d}{3}, \mathrm{~S}_{2}$ for $a=\frac{f+c}{3}$, and $S_{\mathbf{z}}$ for $b=\alpha+d$, as stated before.

Hence we know what relations between the different variable should not exist.

This is all we can get from the function S. For further relations we must look to the function $\mathrm{D}$.

For station I. we have *

$$
\mathrm{D}^{\prime}=\frac{e^{\prime}}{e^{\prime \prime}} \cdot \frac{\mathrm{K}^{\prime \prime}}{\mathrm{R}^{\prime} \mathrm{K}^{\prime}} \cdot \frac{\Delta^{\prime}}{\mu^{\prime} \sqrt{a^{\prime}}},
$$

which, again, with respect to the variations of $\mathrm{K}^{\prime}, \mathbf{R}^{\prime}$, and $\lambda^{\prime}$, may be written in three different forms :-

and

$$
\begin{aligned}
& \mathrm{D}_{1}^{\prime}=\frac{e^{\prime}}{e^{\prime \prime}} \frac{\mathrm{K}^{\prime \prime}}{\overline{\mathrm{R}^{\prime} \mathrm{K}^{\prime}}} \cdot \frac{\lambda^{\prime} v^{\prime} \sqrt{b^{\prime}}}{\mu^{\prime} \sqrt{a^{\prime}}} \delta \mathrm{K}^{\prime}, \\
& \mathrm{D}_{2}^{\prime}=\frac{e^{\prime}}{e^{\prime \prime}} \cdot \frac{\mathrm{K}^{\prime \prime}}{\mathrm{R}^{\prime} \mathrm{K}^{\prime}} \cdot \frac{1}{\mu^{\prime}} \delta \mathrm{R}^{\prime},
\end{aligned}
$$

Considering that

$$
\mathrm{D}_{3}^{\prime}=\frac{e^{\prime}}{\bar{e}^{\prime \prime}} \cdot \frac{\mathrm{K}^{\prime \prime}}{\mathrm{R}^{\prime}} \cdot \frac{\overline{v^{\prime}} \sqrt{b^{\prime}}}{\mu^{\prime} \sqrt{a^{\prime}}} \delta \lambda^{\prime} .
$$

and

$$
\begin{aligned}
\frac{\mathrm{K}^{\prime \prime}}{\mathbf{K}^{\prime}} & =\frac{i+l^{\prime \prime}+\rho^{\prime \prime}}{i+l^{\prime}+\rho^{\prime}}, \\
\mu^{\prime} & =\frac{i}{i+l^{\prime}+\rho^{\prime}}
\end{aligned}
$$

we have

$$
\frac{\mathrm{K}^{\prime \prime}}{\mu^{\prime}}=\mathrm{L}+\rho^{\prime}+\rho^{\prime \prime}+\frac{\left(l^{\prime}+\rho^{\prime}\right)\left(l^{\prime \prime}+\rho^{\prime \prime}\right)}{i},
$$

$$
\begin{aligned}
& \mathrm{D}_{1}^{\prime}=\frac{e^{\prime}}{e^{\prime \prime}} \cdot \frac{i-l^{\prime \prime}+\rho^{\prime \prime}}{i} \cdot \frac{\lambda^{\prime} v^{\prime} \sqrt{b^{\prime}}}{\mathrm{R}^{\prime} \sqrt{a^{\prime}}} \cdot \delta \mathrm{K}^{\prime}, \\
& \mathrm{D}_{2}^{\prime}=\frac{e^{\prime}}{e^{\prime \prime}} \cdot \frac{i+l^{\prime \prime}+\rho^{\prime \prime}}{i} \cdot \frac{1}{\mathrm{R}^{\prime}} \delta \mathrm{R}^{\prime} \\
& \mathrm{D}_{3}^{\prime}=\frac{e^{\prime}}{e^{\prime \prime}}\left\{\mathrm{L}+\rho^{\prime}+\rho^{\prime \prime}+\frac{\left(l^{\prime}+\rho^{\prime}\right)\left(l^{\prime \prime}+\rho^{\prime \prime}\right)}{i}\right\} \frac{v^{\prime} \sqrt{b^{\prime}} \delta \lambda^{\prime} .}{\mathrm{R}^{\prime} \sqrt{a^{\prime}}}
\end{aligned}
$$

* When investigating the minimum absolute magnitude of $\mathbf{S}$, the terms could be taken without an accent, because $S$ contains only terms belonging to the same station. When investigating $\mathrm{D}$ this cannot be done, as $\mathrm{D}$ contains also terms belonging to the other station. 
put

$$
\begin{gathered}
\frac{e^{\prime}}{e^{\prime \prime}}=s \\
\frac{i+l^{\prime \prime}+\rho^{\prime \prime}}{i}=\mathrm{J} \\
\mathrm{L}+\rho^{\prime}+\rho^{\prime \prime}+\frac{\left(l^{\prime}+\rho^{\prime}\right)\left(l^{\prime \prime}+\rho^{\prime \prime}\right)}{i}=\mathrm{T},
\end{gathered}
$$

and

$$
\begin{aligned}
\frac{1}{\mathrm{R}^{\prime} \sqrt{\frac{a^{\prime}}{b^{\prime}}}} & =\frac{1}{\psi^{\prime}} \\
\therefore \mathrm{D}_{1}^{\prime} & =s \mathrm{~J} \lambda^{\prime} v^{\prime} \cdot \frac{1}{\psi^{\prime}} \delta \mathrm{K}^{\prime}, \\
\mathrm{D}_{2}^{\prime} & =s \mathrm{~J} \frac{1}{\mathrm{R}^{\prime}} \delta \mathrm{R}^{\prime}, \\
\mathrm{D}_{3}^{\prime} & =s v^{\prime} \frac{\mathrm{T}}{\psi^{\prime}} \delta \lambda^{\prime} .
\end{aligned}
$$

Now keeping $s, \mathrm{~J}, \lambda^{\prime}$, and $v^{\prime}$ constant, $\mathrm{D}_{1}^{\prime}$ becomes smaller for any given $\delta \mathrm{K}^{\prime}$ the larger $\psi^{\prime}$ is selected; while $\mathrm{D}_{3}^{\prime}$ becomes smaller for any given $\delta \lambda^{\prime}$ the smaller $\frac{\mathrm{T}}{\psi^{\prime}}$ is selected, and $\mathrm{D}_{2}^{\prime}$ becomes smaller the larger $R^{\prime}$ is chosen.

$$
\begin{gathered}
\text { Now } \psi^{\prime}=\mathrm{R}^{\prime} \sqrt{\overline{a^{\prime}}} \text { has a maximum for } a^{\prime}=b^{\prime} ; \text { for } \\
\mathrm{R}^{\prime}=b^{\prime}+\alpha^{\prime}+d^{\prime}=b^{\prime}+\gamma^{\prime} ;
\end{gathered}
$$

and putting $\gamma^{\prime}=l^{\prime} t^{\prime}$, we have

$$
\psi^{\prime}=\left(1+t^{\prime}\right) \sqrt{a^{\prime} b^{\prime}}
$$

which, for $a^{\prime}+b^{\prime}$ and $t^{\prime}$ constant, has clearly a maximum for $a^{\prime}=b^{\prime}$. This proceeding is right, because we take $b^{\prime}$ as the original variable, and vary $a^{\prime}$ and $\gamma^{\prime}$ simultaneously with $b^{\prime}$ in order to keep $t^{\prime}$ and $a^{\prime}+b^{\prime}$ constant; while $J$ and $s$ are independent of $a^{\prime}, b^{\prime}$, and $\gamma^{\prime}$.

In order to be sure that $a^{\prime}=b^{\prime}$ makes also $\mathrm{D}_{3}^{\prime}$ a minimum, we must show that $\mathrm{T}$ keeps constant, i. e. $\rho^{\prime}$ keeps constant when $a^{\prime}$ varies. But $\rho^{\prime}=a^{\prime}+f^{\prime}$; thus we have only to consider $f^{\prime}$ simultaneously variable with $a^{\prime}$, equal and opposite to the variation of $a$, which is allowed. Therefore the condition $a^{\prime}=b^{\prime}$ makes undoubtedly the disturbances $\mathrm{D}_{1}^{\prime}$ and $\mathrm{D}_{3}^{\prime}$ minima; while the disturbance $\mathrm{D}_{2}^{\prime}$, which contains $\mathrm{R}^{\prime}$ in the denominator only, is not affected by this relation, but depends on the absolute value of $b^{\prime}$ only, which should be chosen as large as possible. 
$a=b$ is therefore the second regularity-condition, the fulfilment of which makes the relative disturbance of balance by a variation of $K$ and $\lambda$ as small as possible.

Substituting now $a^{\prime}=b^{\prime}$ in the expression of the D disturbances, and remembering that

we get

$$
\mathrm{R}^{\prime}=\mathrm{K}^{\prime} \text {, }
$$

$$
\begin{aligned}
& \mathrm{D}_{\mathrm{L}}^{\prime}=s \lambda^{\prime} v^{\prime} \frac{\mathrm{J}}{\mathrm{K}^{\prime}} \delta \mathrm{K}^{\prime}, \\
& \mathrm{D}_{2}^{\prime}=s \frac{\mathrm{J}}{\mathrm{K}^{\prime}} \delta \mathrm{R}^{\prime}, \\
& \mathrm{D}_{3}^{\prime}=s v^{\prime} \frac{\mathrm{T}}{\mathrm{K}^{\prime}} \delta \lambda^{\prime} .
\end{aligned}
$$

Thus $\mathrm{D}_{1}^{\prime}$ and $\mathrm{D}_{2}^{\prime}$, for constant $s, \lambda^{\prime}$, and $v^{\prime}$, become smaller the smaller $\frac{J}{\overline{\mathrm{K}}^{\prime}}$ is, while $\mathrm{D}_{3}^{\prime}$ becomes smaller the smaller $\frac{\mathrm{T}}{\mathrm{K}^{\prime}}$ is.

Now remembering that

and

$$
\begin{aligned}
\mathrm{J} & =\frac{i+l^{\prime \prime}+\rho^{\prime \prime}}{i}, \\
\mathrm{~K}^{\prime} & =\frac{\left(l^{\prime \prime}+\rho^{\prime \prime}\right)\left(i+l^{\prime}+\rho^{\prime}\right)+i\left(l^{\prime}+\rho^{\prime}\right)}{i+l^{\prime \prime}+\rho^{\prime \prime}},
\end{aligned}
$$

$$
\begin{aligned}
\mathrm{T} & =\mathrm{L}+\rho^{\prime}+\rho^{\prime \prime}+\frac{\left(l^{\prime}+\rho^{\prime}\right)\left(l^{\prime \prime}+\rho^{\prime \prime}\right)}{i}, \\
\therefore \frac{\mathrm{J}}{\mathrm{K}^{\prime}} & =\frac{\left(i+l^{\prime \prime}+\rho^{\prime \prime}\right)^{2}}{i\left\{\left(l^{\prime \prime}+\rho^{\prime \prime}\right)\left(i+l^{\prime}+\rho^{\prime}\right)+i\left(l^{\prime}+\rho^{\prime}\right)\right\}}, \\
\frac{\mathrm{T}}{\overline{\mathrm{K}}^{\prime}} & =\frac{i+l^{\prime \prime}+\rho^{\prime \prime}}{i}=\frac{1}{\mu^{\prime \prime}} .
\end{aligned}
$$

For a tolerably good line $l^{\prime \prime}+\rho^{\prime \prime}$ as well as $l^{\prime}+\rho^{\prime}$ can be taken as small in comparison with $i$; hence, approximately,

and

$$
\frac{\mathrm{J}}{\mathrm{K}^{\prime}}=\frac{1}{l^{\prime}+l^{\prime \prime}+\rho^{\prime}+\rho^{\prime \prime}}=\frac{1}{\mathrm{~L}+\rho^{\prime}+\rho^{\prime \prime}},
$$

$$
\frac{\mathrm{T}}{\mathrm{K}^{\prime}}=1 \text {, }
$$

from which it follows that also for the compensation method $\rho^{\prime}$ and $\rho^{\prime \prime}$ should be selected as large as possible.

But $\rho=a+f$ does not give a condition besides that we know we should select $a$ and $f$ absolutely not small. 
Further, we see that the disturbance $\mathrm{D}_{3}^{\prime}$ has $v^{\prime}$ for its factor, while $\mathrm{D}_{1}^{\prime}$ has $\lambda^{\prime} v^{\prime}$ for its factor.

Hence for a given $\lambda^{\prime} v^{\prime}$, the best will be to make $v^{\prime}$ as small as possible.

The regularity of the signals is therefore obtained if we fulfil the following conditions in either station :-

$$
\begin{aligned}
& \mathrm{R}=\mathrm{K}, \\
& a=b, \\
& \rho \quad \text { as large as possible, } \\
& v \quad \text { as small as possible. }
\end{aligned}
$$

Knowing this, we may now consider that balance in either station is rigidly obtained, or that

$$
\mathrm{R} \sqrt{a}-\mathrm{K} \lambda v \sqrt{b}=0 \text {; }
$$

but

and

$$
\mathrm{R}=\mathrm{K}
$$

$$
a=b \text {; }
$$

we have

$$
\lambda v=1 \text {. }
$$

The absolute value of $a$ may now be determined by considering that it is advisable to produce the signals in either station in the most economical manner.

Maximum Magnetic Moment.

We have

$$
\begin{aligned}
\mathrm{P}^{\prime} & =\frac{e^{\prime \prime}}{a^{\prime \prime}+f^{\prime \prime}+c^{\prime \prime}} \mu^{\prime} q^{\prime} \sqrt{a^{\prime}}, \\
\mathrm{P}^{\prime \prime} & =\frac{e^{\prime}}{a^{\prime}+f^{\prime}+c^{\prime}} \mu^{\prime \prime} q^{\prime \prime} \sqrt{a^{\prime \prime}} .
\end{aligned}
$$

But

where

$$
\frac{\mu^{\prime}}{a^{\prime \prime}+f^{\prime \prime}+c^{\prime \prime}}=\frac{\mu^{\prime \prime}}{a^{\prime}+f^{\prime}+c^{\prime}}=\frac{i}{\mathrm{Q}}
$$

$$
\begin{aligned}
\mathrm{Q} & =i\left(\mathrm{~L}+\rho^{\prime}+\rho^{\prime \prime}\right)+\left(l^{\prime}+\rho^{\prime}\right)\left(l^{\prime \prime}+\rho^{\prime \prime}\right) ; \\
\therefore \mathrm{P} & =\mathrm{P}^{\prime}+\mathrm{P}^{\prime \prime}=i \frac{e^{\prime \prime} q^{\prime} \sqrt{a^{\prime}}+e^{\prime} q^{\prime \prime} \sqrt{a^{\prime \prime}}}{\mathrm{Q}},
\end{aligned}
$$

which has a maximum for $a^{\prime}$ and $a^{\prime \prime}$ taken as independent variables.

If we, for instances, take $i=\infty$, then

$$
\begin{aligned}
\mathrm{P} & =\frac{e q \sqrt{a}}{\mathrm{~L}+2(a+f)} ; \\
\therefore a & =\frac{\mathrm{L}}{2}+f \text { for a perfect line } ;
\end{aligned}
$$


and by inference

$$
\left.\begin{array}{r}
a^{\prime}=\frac{\mathrm{L}^{\prime}}{2}+f^{\prime}, \\
a^{\prime \prime}=\frac{\mathrm{U}^{\prime \prime}}{2}+f^{\prime \prime}
\end{array}\right\} \text { approximately. }
$$

Now we can decide on the method to be adopted for readjusting balance. On account of the regularity-condition $R=K$, and as both undergo variation, especially $K$, we are obliged to adjust balance in the compensation branch by varying the resistance $d$, and leave the coils or their armatures stationary.

Thus the general solution of the first problem for the compensation method is :

1. Readjustment of balance is to be effected by a variation of resistance in the compensation circuit, and not by a movement of the coils or their armatures. By this adjustment $\mathrm{R}$ is kept equal to $\mathrm{K}$ permanently, no matter in which branch the variation takes place.

2.

$$
\begin{aligned}
\mathbf{f} & =\mathrm{w}+\boldsymbol{\beta} ; \\
\mathrm{a} & =\mathrm{b}=\frac{\mathrm{L}}{\mathbf{2}}+\mathrm{f} ; \\
\mathrm{v} \lambda & =1,
\end{aligned}
$$

$\checkmark$ as small as possible and $\lambda$ as large as possible.

$\beta$ is known from the number and nature of the single cells of which the battery has to consist to produce through the given line (connected up in a circuit like fig. 3) single signals with sufficient strength.

$w$ is known from the absolute largest variation $\beta$ may undergo in time; hence $f$ is determined, and therefore also $a$ and $b$.

Determination of $\lambda$ and $\mathrm{v}$.

We know that $\lambda v=1$, and, further, that $\lambda=\frac{\mathrm{E}}{e}$ should be selected as large as possible or $v$ as small as possible'; but otherwise it appears that no fixed values for $\lambda$ and $v$ can be ascertained. If we, however, consider the nature of the variations of $R$ and $K$ which may disturb the balance, viz. those variations of $R$ and $K$ which are due to unavoidable decrease of the internal resistance of the two batteries by the working currents, it will be seen that a best value of $\lambda$ does exist, and that therefore $v$ also becomes fixed.

Suppose that at a certain moment

$$
\mathrm{R}=\mathrm{K}
$$

is rigidly fulfilled; and remembering that

$$
\begin{aligned}
& \mathrm{R}=b+d+a, \\
& \mathrm{~K}=2(a+f)+\mathrm{L}
\end{aligned}
$$


(for a perfect line, i. e. $i=\infty$ ), and that, further,

$$
a=b
$$

and

we have

$$
f=w+\beta,
$$

$$
d+\alpha=a+2 w+2 \beta+\mathrm{L} \text {. }
$$

Now in this equation suppose every thing constant except $\alpha$ and $\beta$, the internal resistance of the two batteries $\mathrm{E}$ and $e$ respectively. Hence, if we could achieve that

$$
\delta \alpha=2 \delta \beta \text { invariably, }
$$

the variation of the internal resistance of the two batteries would not disturb the equation $R=K$, and therefore also not affect the balance. With absolute certainty we cannot fulfil this desirable relation between the two variations; but with some probability we may. For it is well known that the internal resistance of a galvanic battery decreases in time by the current passing through the battery. Hence, if we suppose that the two batteries consist of identical cells (equal in nature, size, and internal resistance), we may say that the variation of the internal resistance of a single cell by the unit current in the unit of time is the same for both the batteries. Further, if we make the other not improbable supposition that the variation at any one time is proportional to the current which passes at that time, we have

and

$$
\delta \alpha=\epsilon \mathrm{E} \cdot \frac{\mathrm{E}}{\mathrm{R}+\delta \mathrm{R}} \phi^{(t)}=\epsilon \frac{\mathrm{E}^{2}}{\mathrm{R}} \phi^{(t)}
$$

$$
2 \delta \beta=\epsilon e \cdot \frac{e}{\mathrm{~K}+\delta \overline{\mathrm{K}}} \phi^{(t)}=\epsilon \frac{e^{2}}{\overline{\mathrm{K}}} \phi^{(t)},
$$

where $\epsilon$ is the variation of the internal resistance of a single cell in unit of time by unit of current, $\phi^{(t)}$ a certain unknown function of the time which, as the two batteries are working simultaneously, is not required to be known.

Hence, from

and

$$
\delta \alpha=2 \delta \beta
$$

it follows that

$$
\mathrm{K}=\mathrm{R} \text {, }
$$

$$
\lambda=\frac{\mathbf{E}}{\mathbf{e}}=\sqrt{2}
$$

and

$$
v=\frac{r}{q}=\sqrt{\frac{1}{2}}
$$

These values of $\lambda$ and $v$ bring the compensation method, 
with respect to regularity of working, as close to the differential method as is possible for us to do ; for the disturbance of balance in the sending-station by the steady decrease of the internal resistance of the two batteries has now been probably eliminated, which defect is excluded from the other two methods by their own nature. There are then remaining only those variations of the battery-resistance which do not follow the law of steady decrease, but which are more accidental, and make therefore the compensation method still inferior to either the differential or bridge method.

$$
\text { Physical meaning of } \mathrm{v}=\sqrt{\frac{1}{2}} \text {. }
$$

It has been proved that balance in each station is to be established by adjusting resistance, and not by a movement of the coils or their armatures. Hence it will be practical and convenient to coil the two helices above each other and have them acting on one and the same iron core.

Further, as $v=\frac{r}{q}=\sqrt{\frac{1}{2}}$, it follows that the magnetic action of the $a$ coil must be made greater than that of the $b$ coil. Therefore it will be best to coil the helix $b$ on the top of the helix $a$.

Further, the magnetic action of a cylindrical coil of resistance $a$ (in Siemens units) can be expressed as follows-

$$
m=s \sqrt{a} \sqrt{\frac{\mathrm{A} \lambda}{c l}}
$$

where $A$ is half the cross section of the coil (cut by a plane through the axis of the coil) expressed in square millimetres;

$\lambda$ the absolute conductivity of the wire material $\left(\mathrm{H}_{s}=1\right.$ at $\left.0^{\circ} \mathrm{C}.\right)$;

$l$ the length of an average convolution expressed in metres;

$s$ the magnetic force exerted by an average convolution of the coil when the unit of current passes;

$c$ a coefficient representing the manner of coiling.

Hence for the $a$ coil we have

$$
m_{a}=s^{\prime} \sqrt{a} \sqrt{\frac{\hat{A}^{\prime} \lambda^{\prime}}{c^{\prime} l^{\prime}}}=q \sqrt{a},
$$

for the $b$ coil

$$
m_{b}=s^{\prime \prime} \sqrt{b} \sqrt{\frac{\bar{A}^{\prime \prime} \lambda^{\prime \prime}}{c^{\prime \prime} l^{\prime \prime}}}=r \sqrt{b} .
$$

Dividing $m_{b}$ by $m_{a}$, and remembering that by condition $a=b$, and that $\lambda^{\prime}=\lambda^{\prime \prime}, c^{\prime}=c^{\prime \prime}$ by necessity, we have

$$
v=\frac{r}{q}=\frac{s^{\prime \prime}}{s^{\prime}} \sqrt{\frac{\mathrm{A}^{\prime \prime} l^{\prime}}{\mathrm{A}^{\prime} l^{\prime \prime}}}
$$


As we have supposed that the magnetic action of any one cylindrical coil is proportional to the magnetic action* of am average convolution, it is also consistent to put $s^{\prime}=s^{\prime \prime}$, and we have at last

$$
\frac{\mathrm{A}^{\prime \prime} l^{\prime}}{\mathrm{A}^{\prime} l^{\prime \prime}}=\frac{1}{2} \text {. }
$$

If now the two bobbins of the coils $a$ and $b$ are taken of equal length, and if the thickness of the $a$ coil be $d^{\prime}$, the thickness of the $b$ coil $d^{\prime \prime}$, and the diameter of the iron core $2 r^{2}$, we have

$$
\begin{aligned}
\frac{\mathrm{A}^{\prime \prime}}{\mathrm{A}^{\prime}} & =\frac{d^{\prime \prime}}{d^{\prime}}, \\
l^{\prime} & =\left(2 r+d^{\prime}\right) \pi, \\
l^{\prime \prime} & =\left\{2\left(r+d^{\prime}\right)+d^{\prime \prime}\right\} \pi ; \\
\therefore \quad\left(4 r+d^{\prime}\right) d^{\prime \prime} & =2 d^{\prime}\left(r+d^{\prime}\right) .
\end{aligned}
$$

This equation fixes the relative dimensions of the two bobbins and their cores in order to have $v=\sqrt{\frac{1}{2}}$.

Suppose, for instance, we make $d^{\prime}=d^{\prime \prime}$ arbitrarily $\dagger$, we get $2 r=d$; and from it can be easily calculated that the diameter of the wire of the $b$ coil should be about 19 per cent. larger than that of the $a$ coil. The absolute diameter of the wire depends of course on the absolute dimensions of the bobbins, and on the resistance of the line for which the instrument is to be

* Lenz and Jacobi have experimentally proved that, within certain limits, the magnetic force exerted by a convolution on its centre (iron core) is almost independent of the diameter of the convolution. These limits are generally fulfilled in telegraph-construction. Hence the magnetic action of a coil can be put proportional to the magnetic action of one convolution. Theoretically this can of course not be true; for the magnetic force exerted by a convolution necessarily extends on both sides of the plane in which the convolution is situated. Therefore the wider a convolution is, the less of its total force exerted will be made use of for producing magnetism in the iron core, and consequently the force exerted by a convolution on its centre must decrease with the diameter of the convolution. It appears, however, that this decrease is exceedingly slow, and in the present investigation it is considered unnecessary to be taken into account.

+ I have not been able to find anywhere a definite law which connects the diameter of a coil with the diameter of the core acted upon. In Siemens's relay, an instrument so well considered in all its details of construction, the diameter of the coil is about three times the diameter of the core. In the absence of any thing else on the subject I thought myself justified in using this proportion. Hence the substitution of $d^{\prime}=d^{\prime \prime}$, which gives $d=2 r$, or total diameter of the $a$ coil equal to three times the diameter of the iron core. 
used. But this question, although of practical importance, has nothing to do with the Theory of Duplex Telegraphy. This settles the solution of the first problem of the compensation method.

Other methods.-There have been suggested from time to time many other methods of duplex working. On a closer examination it will, however, be found that, as a general rule, they do not differ essentially from the three fundamental methods treated of. I shall therefore dispense with the labour of investigating these derived methods.

In case it should be thought necessary to investigate them, no difficulties ought to be met with, if only the general plan of attacking duplex problems be remembered, viz.:- draw the diagram of the method in its most general form; develop the forces $p, \mathrm{P}$, and $\mathrm{Q}$; from these three forces determine the functions $S$ and $D$; find the relations which must hold between the different variables (resistances and electromotive forces) of which the system consists, in order to make S and D simultaneous minima; consider the question of immmediate balance which determines also the best mode of adjusting balance; consider that the movement of the key must not alter the complex resistance of the station to which the key belongs, $i . e$. that the working of the key must not affect the balance of the distant station; determine the absolute values of the different variables when balance is rigidly fulfilled by considering the question of economy, $i$. e. establish the relations for maxima currents and maxima magnetic moments. Any variables which should then be left indeterminate must be fixed by secondary considerations and by certain practical conditions.

Before comparing quantitatively the efficiency of the three fundamental methods treated of, it is required to solve two questions, viz. the electromotive force required for each duplex method, and the absolute size of the increments of the adjustable resistance.

[To be continued.]

LXIII. Remarks on the Discovery of Gallium. By D. MeNDELEJEF".

TN 1869 I announced the following poriodic law :- "The 1 properties of the simple bodies, as also the properties and constitution of their combinations, are periodic functions of the atomic weights of the elements."

Among the different applications of that law I shall cite only the following :-

1. This law constitutes the basis of a complete systematic classification of the elements.

* Translated from the Comptes Rendus by M, M, Pattison Muir. 